\title{
TTR
}

Traduction, terminologie, re?daction

\section{La traduction journalistique au Québec (1764-1855)}

\section{Ginette Demers}

Volume 6, numéro 1, 1er semestre 1993

\section{L’Histoire en traduction}

URI : https://id.erudit.org/iderudit/037141ar

DOI : https://doi.org/10.7202/037141ar

Aller au sommaire du numéro

\section{Éditeur(s)}

Association canadienne de traductologie

ISSN

0835-8443 (imprimé)

1708-2188 (numérique)

Découvrir la revue

Citer cet article

Demers, G. (1993). La traduction journalistique au Québec (1764-1855). TTR,

6(1), 131-147. https://doi.org/10.7202/037141ar d'utilisation que vous pouvez consulter en ligne.

https://apropos.erudit.org/fr/usagers/politique-dutilisation/ 


\section{La traduction journalistique au Québec (1764-1855)}

\section{Ginette Demers}

Depuis la Conquête, la traduction journalistique joue un rôle considérable au Québec: nos premiers journaux étaient bilingues, et la place importante que tient la traduction dans les journaux unilingues français publiés par la suite est attestée par de nombreux auteurs. ${ }^{1}$ Pourtant, il n'existe pas de véritable étude à ce sujet. Il semblait donc intéressant de se pencher sur ce type de traduction et de tenter de cerner son évolution de même que son influence sur la langue.

\section{Aspects méthodologiques}

Le présent article présente les résultats partiels obtenus à la suite d'une recherche sur la période s'étendant de 1764 à 1855. La

1. Voir ci-dessous ("les Journaux») des exemples pour la première moitié du XIXe siècle; pour la seconde moitié, Pierre-Louis Lapointe, «la Presse québécoise d'expression française à l'heure de la Confédération", la Presse quebécoise et hulloise d'expression française (Hull, Société historique de l'ouest du Québec, 1973), pp. 6, 23; pour le XXe siècle, Benoît (1922), p. 223; Louis Robillard, "le Chef du service des nouvelles", le Devoir, 23 février 1935; Paul Morisset, «la Traduction dans les médias écrits, ou les escaliers roulants de Bombardiern, Traduction et qualité de la langue (Hull, Conseil de la langue française, 1984), p. 41. 
première de ces dates a été choisie parce qu'elle marque l'apparition de la presse périodique au Québec et la seconde, parce qu'elle représente la fin d'une époque: celle des pionniers de la traduction journalistique (voir ci-dessous, «les Journaux»).

Je me suis uniquement intéressée à la traduction de l'anglais au français, dans les journaux axés sur l'information dite "générale». Parmi les nombreux journaux de ce genre publiés au cours de la période d'observation, ce sont The Quebec Gazette / la Gazette de Québec, le Canadien et la Minerve ${ }^{2}$ qui ont été retenus, tant en raison de leur longévité qu'en raison de l'importance que leur accordent les historiens. ${ }^{3}$

Comme le contenu de ces journaux touche plusieurs domaines, j'ai dû restreindre le champ d'étude. À ce stade de la recherche, la réflexion a surtout porté sur les textes à caractère politique. Deux autres domaines, l'actualité internationale et la publicité, seront étudiés ultérieurement. ${ }^{4}$

Je décrirai d'abord brièvement le contexte politique et social de la période observée ainsi que les journaux analysés. J'esquisserai ensuite un portrait des principaux traducteurs de

2. Ainsi, la Gazette de Montréal / The Montreal Gazette a été éliminée parce que, les premières années, la traduction y était effectuée du français à l'anglais et que, par la suite, le contenu était sensiblement le même que celui de la Gazette de Québec.

3. À ce sujet, voir Galarneau (1970), p. 53; Wallot (1973), p. 299; Hare (1977), p. 27; Lemoine (1981), pp. 10-11.

4. Ces trois domaines ont fait l'objet d'un volume important de traduction, et l'espace qu'ils occupent dans les journaux témoigne de l'intérêt qu'ils suscitaient. En effet, $d^{\prime}$ après Hare et Wallot (1983), la répartition de l'espace dans les périodiques d'expression française publiés à Québec, Montréal et TroisRivières entre 1811 et 1820 était la suivante: publicité, $31,62 \%$; international, $21,81 \%$; culturel, $17,45 \%$; politique, $14,83 \%$; social, $8,92 \%$; économie, $3,56 \%$; militaire, $1,01 \%$; religion $0,80 \%$ (p. 90). 
l'époque et donnerai un aperçu de la documentation à laquelle ils avaient accès. J'aborderai, enfin, la question de la qualité de la langue dans les textes traduits.

\section{Le contexte}

Les quarante dernières années du XVIII siècle et la première moitié du XIXe sont caractérisées par de profonds changements politiques, aussi bien au Canada qu'à l'étranger.

En 1763, la Nouvelle France devient une colonie anglaise. De ce fait, elle est soumise aux lois britanniques et à un régime qui ne lui est pas familier. Selon la tradition britannique voulant que le peuple soit éclairé, Murray, le premier gouverneur, profite de l'apparition de la presse périodique, l'année suivante, pour diffuser en anglais et en français les lois, proclamations, ordonnances et avis. Cette pratique sera répétée par ses successeurs de sorte que, jusqu'en 1823, ces textes seront publiés régulièrement dans la Gazette de Québec. A partir de cette date, les textes officiels, de plus en plus nombreux, paraitront dans la Gazette de Québec par autorité, l'ancêtre de la Gazette officielle de Québec.

Parallèlement, les Canadiens français commencent à s'intéresser activement à la politique dès la Conquête. L'Acte de Québec, en 1774, leur permet d'accéder aux fonctions publiques. Â partir de 1791, et ce jusqu'à l'application de la responsabilité ministérielle en 1848, ils lutteront «pour concrétiser les principes du parlementarisme inscrits, selon eux, dans la Constitution" (Wallot, 1973, p. 276).

En 1805, la lutte acharnée entre le "parti anglais" et le "parti français» donne naissance à un journal de combat, le Quebec Daily Mercury. L'année suivante, le parti français riposte en fondant le Canadien. Une interruption de la publication du Canadien en 1825 (elle sera reprise en 1831) amène la fondation de la Minerve, destinée à prendre la relève. 
Sur le plan international, les événements qui ont fait couler le plus d'encre dans les journaux bilingues ou les journaux unilingues français sont la Révolution américaine et les bouleversements politiques qui ont agité la France de 1789 au Second Empire.

Pour ce qui est du commerce, il n'y eut aucun échange direct entre le Canada et la France de 1764 à 1855, en raison des pratiques coloniales de l'époque. Les nouvelles d'Europe venaient donc en grande partie de Londres ou des États-Unis, de sorte qu'elles devaient être traduites. ${ }^{5}$ De plus, l'offre de livres français était assez restreinte (surtout pendant les périodes où l'Angleterre était en guerre avec la France), et les ouvrages étaient chers puisqu'ils devaient être obtenus par l'intermédiaire d'un agent ou d'un libraire en Angleterré.

Hare (1970) évalue à «dix ou douze pour cent» (p. 86) la proportion de la population francophone du Québec qui savait lire et écrire à la fin du XVIII ${ }^{e}$ siècle. Une autre étude, portant sur une période plus vaste (1750-1850), montre que $35 \%$ environ des francophones "savaient signer».? Dans ces conditions, on peut se demander si les traductions figurant dans les journaux ont pu avoir une influence significative sur la langue. Selon plusieurs auteurs, le contenu des journaux était connu d'un nombre de personnes beaucoup plus important que les tirages ne le laissent croire: "Un même journal passe de main à main, suscite des commentaires et des répétitions orales, de sorte que le tirage n'en révèle que bien imparfaitement le rayonnement et l'influence» (Hare et Wallot, 1983, p. 98).

5. Voir Audet (1932), p. 100 et Hare (1977), p. 24.

6. D'après Lemoine (1981), p. 216; Gallichan (1988), p. 117; Lajeunesse (1988), p. 143.

7. Allan Greer, «l'Alphabétisation et son histoire au Québec. État de la question", $l^{\prime}$ Imprime au Quebec. Aspects historiques (18-20 siècles) (Québec, IQRC, 1983), pp. 25-51. 
A ce sujet, il est amusant de constater que Murray avait pris une mesure assez radicale pour assurer la diffusion des textes officiels publiés dans la Gazette de Québec: afin que nul ne puisse plaider l'ignorance de la loi, il obligeait les curés «à se faire envoyer ladite Gazette toutes les semaines» et à lire ces textes à leurs ouailles le dimanche, faute de quoi ils répondraient «à leurs périls» (la Gazette de Québec, 21 février 1765).

Dans la seconde moitié du XIX'e siècle, l'alphabétisation connaîtra une croissance rapide. $\grave{A}$ la suite d'une entente commerciale entre l'Angleterre et la France, les livres importés pourront être obtenus à prix populaire à partir de 1855 . Les échanges culturels entre le Canada et la France, enfin, seront grandement facilités, des relations diplomatiques ayant été établies quelques années plus tard.

\section{Les journaux}

The Quebec Gazette / la Gazette de Québec fut fondée en juin 1764 par William Brown et Thomas Gilmore, de Philadelphie. Ce journal est bilingue jusqu'en 1832. Pendant les dix années suivantes, il y a deux éditions distinctes: pour la première fois, la partie française de la Gazette est rédigée directement en français. A partir de 1842, cependant, seule l'édition anglaise est conservée. En 1874, la Quebec Gazette se fusionne avec le Quebec Chronicle. En 1925, une nouvelle fusion, avec le Quebec Telegraph, donnera naissance au Quebec Chronicle Telegraph.

A la mort de Brown, en 1789, son neveu, John Neilson, hérite de la Gazette. Il administrera le journal, alternativement avec son fils Samuel, jusqu'en 1848. Parmi les rédacteurs, Ronald Macdonald est le plus remarquable.

De 1764 à 1832, les nouvelles internationales occupent une place considérable comparativement aux nouvelles locales. Il en est de même de la publicité, à laquelle est consacrée au moins une page sur quatre. Comme il y a peu de nouvelles de l'étranger pendant l'hiver, on publie également des «pièces en vers et en prose» destinées à plaire «à l'imagination» et à 
instruire «le jugement» (la Gazette de Québec, 21 juin 1764). Sur le plan politique, c'est un journal modéré, sauf de 1834 à 1840, période où la Gazette eporte des attaques très dures contre le parti canadien» (Beaulieu et Hamelin, 1965, p. 212).

Dans ce journal bilingue, tout est traduit sauf certaines annonces - elles coûtent moins cher si elles sont publiées dans une seule langue - et, de façon très générale, les poèmes. Les fondateurs de la Gazette font déjà une distinction entre traduction littéraire et traduction pragmatique, comme le montre l'extrait suivant:

Comme la traduction des vers d'une langue à l'autre exige une veine poétique, on espère que cela servira d'excuse au Public, de ce que les vers cy dessus n'ont été insérés que dans un langage (21 juin 1764).

Le Canadien fut fondé par Pierre Bédard et François Blanchet, tous deux députés. Une première série est publiée de 1806 à 1810, année où «Bédard, Blanchet et J.-F. Taschereau, de même que l'imprimeur, furent arrêtés pour menées séditieuses» (Beaulieu et Hamelin, 1965, p. 179). A cette époque, leurs principaux collaborateurs sont Jacques et Denis-Benjamen Viger ainsi que Jacques Quesnel.

La publication reprend en 1817. Laurent Bédard est alors rédacteur et imprimeur du journal. A la suite d'une autre interruption - de 1819 à 1820 - c'est Flavien Vallerand qui assume ces fonctions jusqu'en 1825; cette année-là, il doit suspendre la publication en raison de difficultés financières. La dernière série commence en 1831 et se termine en 1909. Entre 1831 à 1855, les trois rédacteurs qui se démarquent sont Étienne Parent, Napoléon Aubin et Ronald Macdonald, qui avait aussi été rédacteur à la Gazette de Québec.

Dans le Canadien, les nouvelles internationales occupent une place moindre que dans la Gazette. Des réflexions d'ordre philosophique et social ainsi que des extraits littéraires $y$ paraissent, mais il s'agit avant tout d'un journal de combat dont les objectifs sont de riposter aux attaques du Quebec Daily 
Mercury contre les Canadiens français, d'instruire le peuple «de ses droits constitutionnels et [d'] accélérer la prise de conscience de son existence collective» (Hare et Wallot, 1967, p. 317).

La traduction $y$ joue évidemment un rôle moins considérable que dans les journaux bilingues, mais ce rôle est loin d'être négligeable, puisqu'en plus des nouvelles internationales, des lettres et des articles figurant dans le Mercury y sont traduits de même que des discours des gouverneurs.

La fondation de la Minerve, en 1826, revient à AugustinAlbert Morin, un étudiant en droit âgé de 23 ans. En 1827, Ludger Duvernay achète le journal et l'imprime, mais Morin en reste le directeur politique et le rédacteur. Duverney est emprisonné pour libelle en 1827,1832 et 1838 , mais demeure propriétaire du journal jusqu'à sa mort en 1858. Les rédacteurs les plus renommés de la Minerve sont Léon Gosselin, Raphaël Bellemare et Antoine Gérin-Lajoie.

A l'instar du Canadien, ce journal a comme objectif principal la défense des droits des Canadiens français. Il contient des éditoriaux, des nouvelles d'Europe, des récits à caractère politique, des faits divers et des poèmes. Les textes traduits $y$ sont nombreux, comme en témoigne cette «Lettre aux abonnés» publiée le 17 novembre 1828:

Nous ne saurions trop le répéter à ceux de nos abonnés que nos travaux n'auraient pas entièrement satisfaits, que la publication d'un journal en langue française dans le BasCanada est extrêmement difficile et pénible; l'Éditeur est réduit à traduire laborieusement $d$ 'une langue étrangère presque tous les morceaux qui remplissent son papier [...]. Encore si l'on n'avait à traduire que les nouvelles étrangères, mais les documents authentiques et officiels de notre politique coloniale, tout ce qui peut éclairer le peuple sur ses droits, ses devoirs, ses rapports avec le gouvernement, nous vient $d$ 'une langue étrangère; il faut l'habiller du langage du peuple pour qu'il puisse en profiter. 
La traduction des textes officiels et des résumés des débats que le gouvernement publiait dans la Gazette de Québec était effectuée par des traducteurs officiels. Pour ce qui est des quatre dernières décennies du XVIII ${ }^{e}$ siècle, deux noms ressortent: François-Joseph Cugnet et Joseph-François Perrault.

Horguelin (1977) a déjà tracé un portrait de Cugnet. Qu'il suffise de rappeler que celui-ci, issu d'une famille de robe, était lui-même avocat et que, dès le début du régime anglais, il commença à exercer une carrière de bureaucrate qui allait être brillante. Il fut, en effet, juge, procureur général, grand voyer, traducteur officiel et secrétaire français du gouverneur et du Conseil de Québec ainsi que greffier du papier terrier. La traduction de l'Acte de Québec lui revient.

Connu surtout pour son rôle dans l'éducation, JosephFrançois Perrault fut tout de même traducteur officiel pendant quelques annnées à la fin du XVIII ${ }^{e}$ siècle. Sa traduction la plus importante est sans contredit celle de la Lex Parliamentaria: Traité de la loi et coutumes des Parlements, publiée en 1803, qui fut un ouvrage fondamental pour les députés de l'époque.

Perrault présente sa traduction sous la forme d'une lettre du traducteur à l'orateur de l'Assemblée. Après avoir loué le gouvernement, il «s'excuse d'avoir utilisé certains mots anglais consacrés, sans équivalents en français: en général, il s'est efforcé d'écrire une langue pure...» (Hare et Wallot, 1967, pp. 49-50).

En 1806, il publie son Dictionnaire portatif et abrégé des loix et règles du Parlement provincial du Bas Canada. Dans la préface, il s'adresse à tous ses compatriotes et indique que l'ouvrage est destiné à faciliter leur connaissance des lois constitutionnelles. 
Parmi les traducteurs officiels de la première moitié du XIXe siècle, citons Philippe Aubert de Gaspé, Guillaume Lévesque $^{8}$ et François-Xavier Garneau. ${ }^{9}$

Pour ce qui est des textes non officiels, les propriétaires de la Gazette de Québec avaient leurs propres traducteurs. Ainsi, Daniel Gallway, en 1765, «retirait 75 livres par année» (Audet, 1932, p. 105). Gallway était également interprète. En outre, il faisait du travail à la pige, comme le montre cette offre de services, la première qu'un traducteur ait fait paraître dans un journal québécois:

Le sieur Galwey interprète constitué pour les cours de judicateures qui doivent être établies sous peu dans la province de Québec [...] fait des interprettations oralles, et traduit toutes pièces écrites ou imprimées [...] N.B. Il traduit des lettres, comptes, \&c. de messieurs les négocians avec tout le soin et l'expédition possible, et avec la plus grande exactitude à garder leurs secrets inviolables (la Gazette de Québec, 12 juillet 1764).

William Vandenvelden, d'origine allemande, était traducteur à la Gazette en 1782. Il était aussi arpenteur et dispensait des cours d'arpentage, de mathématiques et de français. Devenu imprimeur en 1793, il fonda un hebdomadaire bilingue, le Cours du tems / Times, qui ne parut que jusqu'à l'été 1795. Il exerça son métier d'imprimeur jusqu'en 1798, puis se consacra à l'arpentage jusqu'à sa mort en 1809.

De 1808 à 1819, la traduction des articles de la Gazette est effectuée par Jean-Antoine Bouthillier. Son salaire n'est pas beaucoup plus élevé que celui que touchait Gallway 43 ans plus

8. Jacques Gouin (1977) offre un aperçu de la vie mouvementée de ce traducteur.

9. Au sujet des activités de traducteur de François-Xavier Garneau, voir Marc Lebel, «François-Xavier Garneau traducteur». Meta, XXII(1), pp. 33-36. 
tôt, puisqu'il n'atteignait que 100 livres par année. ${ }^{10}$ Bouthillier avait fait ses études au collège Saint-Raphaël et était ensuite devenu arpenteur. Il obtint le poste de greffier adjoint en 1828, fut nommé juge de paix en 1830 et cumula les deux fonctions jusqu'à sa mort en 1835 .

Il ne semble pas $y$ avoir eu de traducteurs "professionnels" au Canadien, ni à la Minerve, du moins avant les années 1840. Comme le fait remarquer Lemoine (1981), «les industries de l'imprimerie, du journal et de la librairie ne font souvent qu'un seul et même commerce. Il n'est pas rentable a l'époque d'exercer une seule de ces activités; pour vivre, il faut savoir combiner avec ingéniosité les métiers d'imprimeur, d'éditeur, de rédacteur, de traducteur, de libraire et de journaliste" (pp. 15-16). A ces fonctions, bon nombre des rédacteurs du Canadien et de la Minerve ajoutaient celle de député. Certains d'entre eux avaient séjourné en Angleterre, d'autres aux États-Unis, de sorte qu'ils peuvent fort bien avoir joué le rôle de traducteurs.

De 1845 à 1847, Antoine Gérin-Lajoie travaille à la Minerve comme correcteur d'épreuves, traducteur et rédacteur. II deviendra traducteur à l'Assemblée législative en 1852 et, en 1854, présentera un projet de réorganisation des bureaux de la traduction. Ce projet sera accepté, et, pendant trois quarts de siècle, cette réorganisation du personnel ne sera à peu près pas modifiée.

10. À titre de comparaison, le salaire des traducteurs officiels, à la même époque, se situait entre 200 et 300 livres (d'après Gilles Paquet et Jean-Pierre Wallot, "la Liste civile du Bas-Canada (1794-1812): un essai d'économie historique", Revue d'histoire de l'Amérique française, XXIV(1), 1970, p. 19). 


\section{La documentation}

Comme les livres importés de France étaient chers et relativement rares et que la production locale était peu abondante ${ }^{11}$, on peut s'interroger sur les outils dont disposait le traducteur. Quelques études nous éclairent à ce sujet.

Lemoine (1981) estime à 6366 le nombre de livres français à vendre ayant fait l'objet d'une annonce dans la Gazette de Québec ou dans le Canadien entre 1764 et 1839. Dans la catégorie «Belles-Lettres», le nombre d'ouvrages français est de 1806 et comprend surtout des livres de poésie et des romans. Les grammaires françaises sont peu nombreuses ( 3 titres). L'auteur souligne que «les dictionnaires les plus nombreux sont surtout en langue anglaise» (p. 212). Les dictionnaires généraux en langue française comptent pour sept titres seulement. C'est peu. Toutefois, les traducteurs pouvaient se tourner vers les bibliothèques.

La bibliothèque du séminaire de Québec, en effet, contenait 5000 livres en 1782. La Bibliothèque de Québec, fondée en 1779 , était à l'origine "une bibliothèque collective de souscription comme il en existait en Grande-Bretagne et dans les colonies américaines depuis le milieu du siècle» (Gallichan, 1988, p. 116). Elle devint cependant accessible aux parlementaires a partir de 1792. A cette époque, elle contenait quelque 2500 ouvrages, dont la moitié environ étaient en français. La Bibliothèque parlementaire, créée en 1802 , ne possédait, en 1811, que 92 livres en français. À partir de 1833, Étienne Parent, qui avait été nommé bibliothécaire de l'Assemblée, voulut lui donner le calibre d'une bibliothèque nationale. Vers le milieu du siècle, «Exception faite des catégories de droit parlementaire et de droit anglais, il y avait des livres français dans toutes les classes de la collection de la Bibliothèque parlementaire du Bas-Canada" (ibid. p. 124).

11. Voir Hare et Wallot (1983), pp. 93-95. 
En outre, Gallichan (1988) estime que certains des "députés, juristes, médecins, nọtaires, journalistes et imprimeurs étaient aussi des bibliophiles avertis, propriétaires de riches collections" (p. 119). Galarneau (1970) est d'avis, également, que la plupart des auteurs cités par des périodiques comme la Gazette de Québec et le Canadien "démontrent à souhait que les rédacteurspropriétaires possèdent de bonnes bibliothèques, fournies d'auteurs latins, anglais et français...» (p. 154). Il semble donc que les traducteurs de la première heure n'aient pas été aussi démunis qu'on aurait pu le croire.

\section{La qualité des traductions}

À une ou deux exceptions près, les opinions sur la qualité des traductions publiées dans les journaux de 1764 à 1855 ne sont guère flatteuses. Déjà, en 1773, Cugnet adresse aux imprimeurs de la Gazette de Québec une version «intelligible» d'un texte officiel paru dans ce journal et dont il juge la traduction incorrecte. Cugnet fait tout de même preuve d'indulgence envers son collègue puisqu'il inscrit entre parenthèses: «sans doute parce que vous n'avez pas donné au traducteur le temps de la travailler...» (cité par Horguelin (1977), p. 24).

Deux siècles plus tard, Gouin (1977) porte le jugement suivant: «Au début, c'est-à-dire de 1764 à 1789, la traduction officielle était excellente. Après 1789, et cela jusque vers les années 1850 , la traduction se détériore, sans doute parce qu'elle est confiée de plus en plus à des gens de formation anglaise» ( $p$. 29). Daviault (1944), cependant, est moins enthousiaste au sujet des premières années. D'après lui, Cugnet était «un bon traducteur moyen [...] il a un style qui se ressent de l'influence de l'anglais (p. 23). Dulong (1967) estime, quant à lui, que l'administration utilise dès le début uun français de traduction farci d'anglicismes, gauchi par une syntaxe étrangère» (p. 10) tandis que Benoît (1922) va jusqu'à qualifier Cugnet de "grand massacreur de la langue française» (p. 264), sans, toutefois, étayer cette affirmation par des exemples. 
Pour ce qui est des autres types de textes figurant dans la Gazette de Québec, Horguelin (1977) n'est pas tendre: «William Brown et Thomas Gilmore étaient sans doute animés de bonnes intentions, mais ils allaient avoir le douteux honneur de susciter une nouvelle "profession", celle de traducteur improvisé, et de créer - avant le mot - une nouvelle langue: le franglais» (p. 20). De Lagrave et Ruelland (1989) considèrent, pour leur part, que "la partie française de la feuille ne renferme que de mauvaises traductions des textes anglais publiés en regard" (Avant-propos). D'après Groulx (1960), enfin, les quarante dernières années du XVIII $e^{e}$ siècle ont été stériles sur le plan intellectuel. Il ajoute qu'«un journal symbolise ces temps de misère, la Gazette de Québec, [...] qui fonde à la fois au pays et la légende du patois canadien et le français bâtard de la traduction...» (p. 78).

Une seule opinion favorable a été repérée. Elle porte sur la troisième décennie du XIXe siècle, époque où Ronald Macdonald était rédacteur en chef de la Gazette: «La justesse de pensée, l'exactitude du style, [...] la bonne tenue et l'élégance des traductions ont fait de la Gazette de Québec un journal lu et estimé par le clergé et les classes instruites" (Saint-Pierre, 1985, p. 594).

Dans le cas du Canadien et de la Mineroe, une seule critique également a été relevée, mais elle est loin d'être positive. Benoît (1922) estime, en effet, que la traduction "joue un rôle effroyable» (p. 258) et que ces journaux «torturent la langue française pour se conformer à un texte anglais» (pp. 259-260). Il ajoute que la traduction explique pourquoi les premiers rédacteurs et collaborateurs du Canadien "n'ont jamais appris à écrire convenablement» (p. 259).

Seule une étude approfondie permettra de déterminer avec exactitude la qualité des traductions dans les trois journaux étudiés. Il est difficile, en effet, de faire la distinction entre «les tournures et termes devenus archaïques, $d$ 'une part, et les erreurs de traduction proprement dites - maladresses, calques et anglicismes - d'autre part» (Horguelin, 1977, p. 19). Effectivement, certains des mots cités comme exemples $\mathrm{d}$ 'anglicismes (indéfatigable, résigner (un emploi), judicature, 
notifier, etc.) apparaissent dans le Dictionnaire de la langue française $d u X V I^{e}$ siècle. D'autres, tel packet boat (devenu paquebot), sont attestés en France au XVIII ${ }^{e}$ siècle (v. Le Petit Robert, 1990, p. 1352).

Les habitudes «traductionnelles» peuvent aussi être différentes d'une époque à l'autre. Ainsi, Horguelin (1977) semble reprocher aux premiers traducteurs leur «tendance à la francisation" (p. 19). Il est vrai que les noms de personnes, les noms de villes et les noms de bateaux sont systématiquement traduits: James Murray devient Jacques Murray ou même M. de Murray, New York devient la Nouvelle York et Brig Naney, le Brigantin La Nannon. Cependant, cette pratique était courante sous le régime français. Le Père Sixte Letac, par exemple, dans son Histoire chronologique de la Nouvelle France, publiée en 1688, traduit les noms propres (Kirk est Quer ou le général de Quer) «suivant l'usage [...] presque général du temps» (Réveillaud, 1980, p. 143, note 1 ).

La traduction massive des textes officiels n'a pu qu'avoir des incidences sur la langue politique au Québec. Par contre, il ne faut pas conclure que toute la terminologie française utilisée à l'Assemblée était l'œuvre des traducteurs. En France, en effet, le vocabulaire parlementaire avait été emprunté à l'anglais entre la fin du XVII ${ }^{e}$ siècle et la fin du XVIII ${ }^{e}$. D'après Hare (1970), l'attestation des termes ajourner, amendement, bill, quorum, warrant, entre autres, remonte respectivement a 1662, 1789, 1669, 1672 et $1704 .^{12}$ Ils devaient donc être connus au Québec à l'époque qui nous intéresse.

Il reste que la Chambre d'assemblée du Bas-Canada ressemblait de beaucoup plus près au Parlement de Londres que les institutions françaises. C'est pourquoi une partie de la terminologie parlementaire et électorale - minutes, constituant,

12. John Hare fournit une liste exhaustive des termes politiques relevés dans les journaux québécois publiés de 1784 à 1812 (1970, pp. 258-522). 
drawback, hustings, indenture, par exemple - a été empruntée directement.

Hare (1970) note également des calques: «emporter dans l'affirmative (in the affirmative), les argents non appropriés (nonappropriated), commettre le bill à un comité (commit a bill) [...] la passation $d^{\prime} u n$ acte (the passation of an act), un proviso à proposer sur une question (a proviso...)» (p. 121).

\section{Conclusion}

La recherche a permis de constater qu'un bon nombre des traducteurs de la période 1764-1855 étaient des hommes cultivés et que certains d'entre eux se sont même illustrés dans le domaine des lettres à leur époque. Ils avaient également accès à une documentation plus abondante qu'on ne le croit généralement. Bien que le contact quotidien avec l'anglais et la contrainte de temps reliée a la traduction journalistique aient certainement eu une influence sur la qualité des textes traduits, il semble peu probable que les traducteurs aient tous été également mauvais. Á la suite d'une comparaison entre certaines des traductions figurant dans les journaux étudiés et des textes publiés dans des journaux français, il serait sans doute possible de faire la part entre le vocabulaire bien français et les anglicismes ainsi qu'entre les tournures désuètes et les calques. Une autre recherche intéressante consisterait à comparer des textes traduits apparaissant dans la Gazette de Québec avec des textes rédigés spontanément en français dans la Gazette de Montréal à la même époque ${ }^{13}$, puisqu'elle permettrait d'évaluer, dans une certaine mesure, la qualité des traductions.

Université Laval

13. En effet, selon Galarneau (1970), le fait que les articles du début aient été rédigés directement en français «explique que la qualité du français soit meilleure dans la Gazette de Montréal que dans celle de Québec» (p. 52). 


\section{Références}

AUDET, Francis-J. (1932). «William Brown (1737-1789). Premier imprimeur, journaliste et libraire de Québec. Sa vie et ses œuvres». Mémoires de la Société royale du Canada, série 3, 26, pp. 97-112.

BEAULIEU, André et Jean HAMELIN (1965). Les Journaux du Québec de 1764 à 1964. Québec / Paris, Presses de l'Université Laval / Librairie Armand Colin.

BENOÎT, R.-Albert (1922). «L'Influence de la traduction sur notre parler». Le Canada français, VIII(4), pp. 253-271.

DAVIAULT, Pierre (1944). "Traducteurs et traduction au Canada». Mémoires de la Société royale du Canada, série 3, 38, pp. 67-87.

DE LAGRAVE, Jean-Paul et Jacques G. RUELLAND (1989). Valentin Jautard 1736-1787. Sainte-Foy, Le Griffon d'argile.

DULONG, Gaston (1967). L'Anglicisme au Canada français. Paris / Québec, Librairie C. Klincksieck / Les Presses de l'Université Laval.

GALARNEAU, Claude (1970). La France devant l'opinion canadienne (1760-1815). Québec / Paris, Presses de l'Université Laval / Librairie Armand Colin.

GALLICHAN, Gilles (1988). «Le Livre français au Parlement du Bas-Canada». Livre et lecture au Québec (1800-1850). Dir. Claude Galarneau et Maurice Lemire. Québec, IQRC, pp. 115-131.

GOUIN, Jacques (1977). «La Traduction au Canada de 1771 a 1867\%. Meta, XXII(1), pp. 26-32.

GROULX, Lionel (1960). Histoire du Canada français depuis la découverte, tome II. Montréal, Fides. 
HARE, John (1970). Lexicologie politique du Canada français. Thèse de Ph. D. Québec, Université Laval.

(1977). La Pensée socio-politique au Québec. 1784-1812. Ottawa, Éditions de l'Université d'Ottawa.

HARE, John et Jean-Pierre WALLOT (1967). Les Imprimés dans le Bas-Canada 1801-1840, vol. 1. Montréal, Presses de l'Université de Montréal.

(1983). Les Imprimés au Québec (1760-1820). Dir. Yvan Lamonde. Québec, IQRC, pp. 77-125.

HORGUELIN, Paul A. (1977). «Les Premiers traducteurs (17601791)». Meta, XXII(1), pp. 15-25.

HUGUET, Edmond (1973). Dictionnaire de la langue française du $X V I^{r}$ siècle. Paris, Didier.

LAJEUNESSE, Marcel (1983). «Le Livre dans les échanges sulpiciens Paris-Montréal au cours de la première moitié du XIX' siècle». Livre et lecture au Québec. Dir. Claude Galarneau et Maurice Lemire. Québec, IQRC.

LEMOINE, Réjean (1981). Le Marché du livre à Québec 1764-1839. Thèse de M. A. Québec, Université Laval.

La Minerve

The Quebec Gazette / la Gazette de Québec

RÉVEILLAUD, E. (1980). Histoire chronologique de la NouvelleFrance ou Canada par le Père Sixte Le Tac, Récollect. Leméac.

ROBERT, Paul (1990). Le Petit Robert 1. Paris, Société du Nouveau Littré.

SAINT-PIERRE, Jocelyn (1985). «Macdonald, Ronald». Dictionnaire biographique du Canada, vol. VIII. 